\title{
An interacting and non-interacting two-fluid dark energy models in FRW universe with time dependent deceleration parameter.
}

\begin{abstract}
We study the evolution of the dark energy parameter in a spatially homogeneous and isotropic FRW space-time filled with barotropic fluid and dark energy by considering a time dependent deceleration parameter. Two cases are discussed when the dark energy is minimally coupled to the perfect fluid as well as direct interaction with it. It is concluded that in both non-interacting and interacting cases only open and flat universes cross the phantom region. We find that during the evolution of the universe, the equation of state $(E o S)$ for dark energy $\omega \mathrm{D}$ changes from $\omega \mathrm{D}>-1$ to $\omega \mathrm{D}<-1$, which is consistent with recent observations. The cosmic jerk parameter in our derived models is also found to be in good agreement with the recent data of astrophysical observations.
\end{abstract}

Keyword: Dark energy; FRW cosmology; Deceleration parameter. 